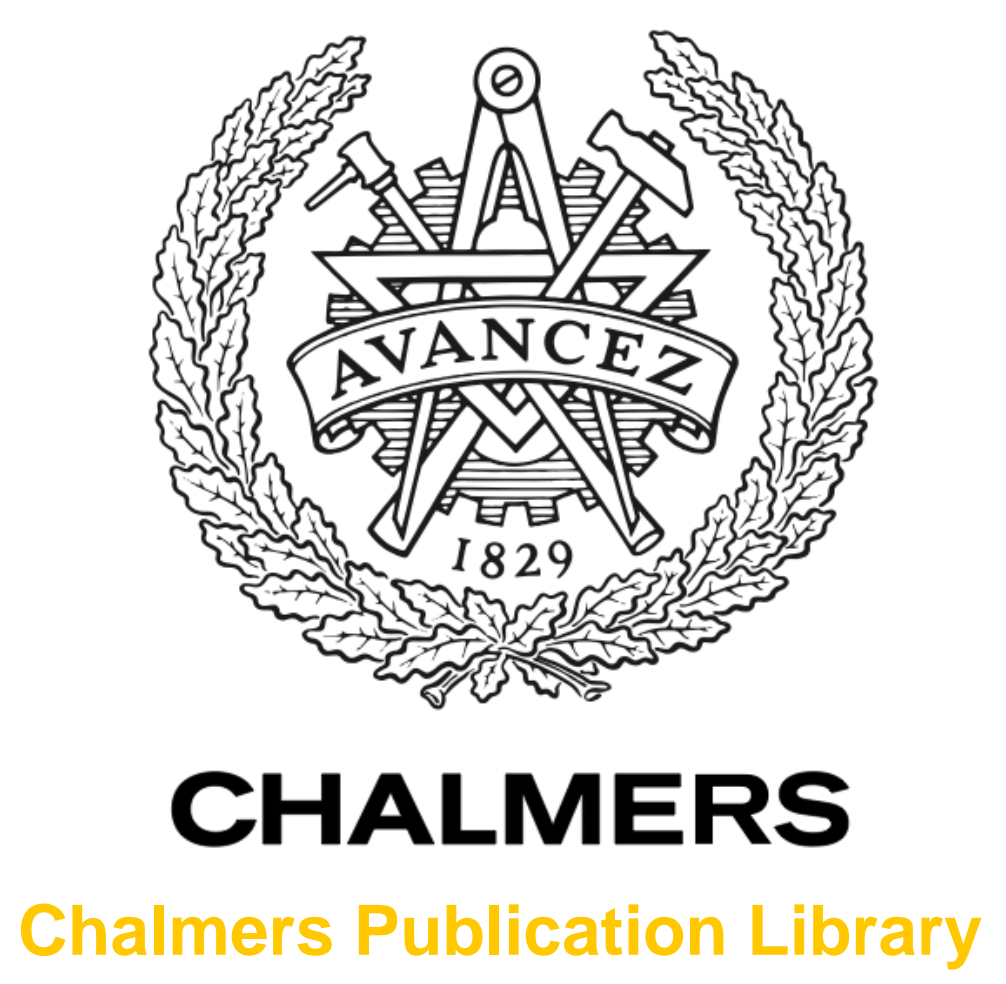

\title{
Solvation structure in dilute to highly concentrated electrolytes for lithium-ion and sodium-ion batteries
}

This document has been downloaded from Chalmers Publication Library (CPL). It is the author's version of a work that was accepted for publication in:

Electrochimica Acta (ISSN: 0013-4686)

Citation for the published paper:

Flores, E. ; Åvall, G. ; Jeschke, S. et al. (2017) "Solvation structure in dilute to highly

concentrated electrolytes for lithium-ion and sodium-ion batteries". Electrochimica Acta, vol. 233 pp. 134-141.

http://dx.doi.org/10.1016/j.electacta.2017.03.031

Downloaded from: http://publications.lib.chalmers.se/publication/249138

Notice: Changes introduced as a result of publishing processes such as copy-editing and formatting may not be reflected in this document. For a definitive version of this work, please refer to the published source. Please note that access to the published version might require a subscription.

Chalmers Publication Library (CPL) offers the possibility of retrieving research publications produced at Chalmers University of Technology. It covers all types of publications: articles, dissertations, licentiate theses, masters theses, conference papers, reports etc. Since 2006 it is the official tool for Chalmers official publication statistics. To ensure that Chalmers research results are disseminated as widely as possible, an Open Access Policy has been adopted.

The CPL service is administrated and maintained by Chalmers Library. 
Solvation structure in dilute to highly concentrated electrolytes for lithium-ion and sodium-ion batteries

\author{
Eibar Flores $^{a, b}$, Gustav Åvall ${ }^{a}$, Steffen Jeschke ${ }^{a}$ and Patrik Johansson ${ }^{a}$ \\ a Department of Physics, Chalmers University of Technology, SE-412 96 Gothenburg, Sweden \\ b Present address: Electrochemistry Laboratory, Paul Scherrer Institut, $\mathrm{CH}-5232$ Villigen PSI, \\ Switzerland
}

Corresponding author: Gustav Åvall: gustav.avall@chalmers.se

\begin{abstract}
The solvation structure of several lithium and sodium based electrolytes are explored as a function of salt concentration over a wide range via a detailed PM7 computational study. The cation coordination shells are found to be well-defined and solvent rich for dilute electrolytes, while disordered and anion rich for the more concentrated electrolytes. The Nabased electrolytes display larger cation coordination shells with a more pronounced presence of fluorine as compared to the Li-based electrolytes. The origins of the structural differences are discussed as well as their consequences for properties of battery electrolytes and battery usage - especially targeting the current large interest in highly concentrated electrolytes.
\end{abstract}

Keywords: highly concentrated electrolytes, solvation structure, lithium ion batteries, sodium ion batteries. 


\section{Introduction}

In a world with a growing urge for electrical energy storage, advances in battery technology are needed to improve the attractiveness for large-scale storage and electrical vehicles (EVs). The limitations with respect to stability, energy and power density needs to be addressed in order to better meet the safety, autonomy, and power standards and improvements required [1][2]. Lithium-ion batteries (LIBs) represent one of the most promising technologies, readily used already today, but recently also the prospect of sodium-ion batteries (SIBs) has re-emerged and grown into an active research topic. The cost of sodium is more than an order of magnitude lower than lithium and sodium is more than three orders of magnitude more abundant, while at the same time SIBs promise a similar level of performance as LIBs [3-7], rendering them future candidates for grid storage [8]. Moreover, the possibility to replace the Cu current collector in LIBs for Al current collectors at both sides in SIBs could push the energy densities further and again lower cost [6,9]. However, the similarities are more plentiful than the differences as for LIBs vs. SIBs.

For both LIBs and SIBs the electrolyte is a key component; e.g. the charge carrier concentration and the ion mobility are both crucial for the power rates as well as overall functionality. The standard LIB electrolyte is today based on $1.0 \mathrm{M} \mathrm{LiPF}_{6}$ in mixtures of organic carbonates (propylene carbonate (PC), ethylene carbonate (EC) and dimethyl carbonate (DMC)) with similar salts and solvents being investigated for SIBs $[1,10]$. Future LIBs/SIBs, however, will require novel electrolytes displaying i) wider electrochemical stability windows to allow cycling of high energy capacity cathodes and especially at higher voltages e.g. up to $5 \mathrm{~V} v$ s. $\mathrm{Li}^{+} / \mathrm{Li}^{\circ}$ [11], ii) compatibility with these and other new electrode chemistries [5], iii) better thermal stability to withstand wider operational temperatures 
[12], and iv) surface and bulk structures and dynamics that allow for faster charge/discharge, i.e., cation desolvation and solvation [13].

Another important aspect is safety, which is mostly addressed by using polymer electrolytes to reduce/eliminate the risk of leakage of flammable liquids. However, polymer electrolytes still suffer from rather poor room-temperature conductivity [14,15]. Recently another concept has emerged; non-aqueous electrolytes with salt concentrations significantly above 1.0 $\mathrm{M}$ have been reported to exhibit remarkable properties including non-volatility, raising the concept of super-concentration (up to ca. $5 \mathrm{M}$ or even above) as an interesting strategy [16]. Reversible LIB operation using both graphite and lithium metal anodes, and with enhanced kinetics have been demonstrated for a number of salts and solvents $[17,18]$. These improvements are attributed to a unique electrolyte structure that promotes an effective electrode passivation $[13,18]$, prevents solvent co-intercalation into graphite $[13,17,19]$, and facilitates (de)lithiation via a different lithium de-solvation mechanism as compared to "conventional" electrolytes [19-21]. The extensive ion-ion interactions are also inferred to be the reason for reduced volatility and hence increased thermal stability and also prevent polysulfide dissolution in the case of Li-S batteries [18,22]. Furthermore, a sharp increase in alkali cation transference number have been observed in ionic liquid based electrolytes, indicating that the transport mechanism is altered at elevated concentrations [23-25], and indeed an increase in lithium transference number was also observed in [18].

As solvents, both acetonitrile (ACN) and PC have recently been shown to allow for a reversible lithium intercalation in graphite only at high salt concentrations $[12,25]$. ACN possesses a dielectric constant of $\varepsilon_{r}=37$, a high oxidation stability [21], and is a rather small and well-studied molecule with respect to both liquid structure and ion solvation [27-29]. As 
for PC, it remains liquid over a much larger temperature range than the often used cosolvent $\mathrm{EC}$ and has a higher dielectric constant compared to $\mathrm{ACN}\left(\varepsilon_{\mathrm{r}}=65\right)$, allowing for a larger degree of salt dissociation enabling higher concentrations. Altogether this makes PC a solvent of interest for highly concentrated electrolytes $[9,25]$.

An understanding of how these solvents and resulting electrolytes behave at the molecular level is needed for any rational improvement. Locally the electrolyte structure can be described in terms of $i$ ) the various solvates formed upon salt dissolution, i.e., solvent separated ion pairs (SSIPs), contact ion pairs (CIPs), aggregates (AGG) [27], and ii) the cation coordination, primarily characterized by the cation-solvent and cation-anion distances and angles. This can either be done experimentally or computationally, and we will here advance the latter approach.

Indeed, the molecular level details of highly concentrated electrolytes have been addressed by several computational studies. Molecular dynamics (MD) simulations have been used to generate a large amount of configurations, thus allowing statistics on solvates as functions of salt concentration and anion type and linking these to phase behaviour $[27,28]$ and transport properties [30]. However, the (classical) force fields used may fail in providing a proper description of highly concentrated electrolytes as the interactions become very nonstandard. Density functional theory (DFT) in principle allows for an accurate description of the (electronic) structure, but as the computational cost scales rapidly with system size, this severely limits the possibilities of modelling any large ensembles and restricts the number of configurations. Therefore, DFT has so far been applied to optimize and validate MD force fields [22] and only recently to reveal the shift of the location of the electrolytes' LUMO from solvent to anion - at high salt concentrations, the latter connected to the improved 
salt-based SEI with better passivation properties [31]. While combining MD with DFT allows for moderate ensemble sizes, the reduced number of configurations will e.g. produce radial distribution functions (RDFs) with a limited spatial resolution [31], and thus the fine structure information of the coordination environment is lost.

As a remedy, we here study the equilibrium configurations from a significant sampling of large ensembles obtained using semi-empirical quantum mechanical calculations. The Parametrized Method 7 (PM7) [32] is a Hartree-Fock based method with significantly improved descriptions of non-covalent interactions such as dispersion, hydrogen bonds and halogen bonds as compared to e.g. PM3 and PM6 [32,33]. By analysing descriptors such as radial distribution functions (RDFs), geometry, and topology, the methodological drawbacks of PM7 tend to vanish, giving results of similar quality as much more expensive DFT methods [34-36], why it also has been used for computations of $\mathrm{Li}^{+}$-solvent cluster dynamics $[37,38]$. In this study we present PM7 calculations on lithium and sodium hexafluorophosphate (LiPF 6 and $\mathrm{NaPF}_{6}$ ) in $\mathrm{PC}$ and $\mathrm{ACN}$ at different salt-to-solvent ratios covering dilute, concentrated, and highly concentrated electrolytes.

\section{Computational}

A set of systems of $\mathrm{LiPF}_{6} / \mathrm{NaPF}_{6}$ in $\mathrm{ACN}$ with different salt-to-solvent ratios; 1:20, 1:16, 1:12, 1:6, 1:4, 2:5, and 4:7, were all built using the software Avogadro [39]. The ratios/concentrations were chosen to span the range from "dilute" via concentrated to highly concentrated electrolytes as addressed and classified by Seo et al. $[18,28]$. Similar molecular ensembles were created for $\mathrm{LiPF}_{6} / \mathrm{NaPF}_{6}$ in PC; 1:12, 1:11, 1:10, 1:8, 1:7, 1:6, 1:5, $1: 4$, and 2:5. These ensembles were made with a racemic mixture of $R-P C$ and $S-P C$. The 
explored molar ratios approximately correspond to 1.0-5.0 M for ACN [30], and for PC ca. 1.0-3.0 M.

The ensembles were subsequently divided into three categories; dilute, concentrated, and highly concentrated. For $\mathrm{ACN}$, the ensembles with molar ratios 1:20, 1:16 and 1:12 where designated as dilute, 1:6 and 1:4 as concentrated, and 2:5 and 4:7 as highly concentrated systems, respectively, and for PC the molar ratios $1: 12$ and $1: 11$ as dilute, $1: 10,1: 8,1: 7,1: 6$ and $1: 5$ as concentrated, and 1:4 and 2:5 as highly concentrated systems, respectively. These three concentration systems/ranges were analysed for the influence of the cation, solvent, and salt concentration on the electrolyte structure.

Geometry optimizations were performed in MOPAC2012 [40,41] using PM7 with an implicit solvent applied via Andreas Klamt's COSMO solvation model with $\varepsilon_{\mathrm{r}}=36.64$ for ACN and $\varepsilon_{\mathrm{r}}=64.92$ for $\mathrm{PC}$, using a radius of $0.18 \mathrm{~nm}$ [10]. Furthermore, frequency calculations were performed and only configurations corresponding to local minima were kept. For the dilute electrolytes 30 cations (extracted from 11 stable configurations) where studied in the ACN based electrolytes and 16 (16 configurations) in the PC, for the concentrated 43 (15 configurations) in ACN and 62 (54 configurations) in PC, and for the highly concentrated 50 (11 configurations) in ACN and 48 (20 configurations) in PC. For the smaller clusters (31 atoms, excluding the hydrogen atoms) stable structures were obtained in less than a minute, while for the larger (272 atoms, excluding the hydrogen atoms) a few hours were needed using an Intel ${ }^{\circledR}$ CORE $^{\mathrm{TM}}$ i7 processor with $16 \mathrm{~GB}$ of RAM.

The RDFs $g(r)$ were explored to obtain the interatomic distances between the electrolyte species. The RDFs are defined as the number $n(r)$ of B atoms located at a distance $r$ from a central atom $A$ within a shell of volume $4 \pi r^{2} \Delta r$ : 


$$
g(r)=n(r) / 4 \pi r^{2} \Delta r
$$

RDFs were calculated for all geometries and then combined to form a single RDF for each of the categories and choice of cation. The first peak in the RDFs was integrated to yield the coordination number $(\mathrm{CN})$. As several ensembles were used to create the RDF the variance

$$
\operatorname{Var}(\mathrm{CN})=\sum_{i}\left(\mathrm{CN}_{i}-\mu\right)^{2} / N
$$

and the standard error

$$
\bar{\sigma}=\sigma / \sqrt{N}
$$

were both calculated, where $\mathrm{CN}_{\mathrm{i}}$ is the coordination number of cation $i, N$ the total number of cations present in all the ensembles belonging to the category, $\mu$ is the mean $\mathrm{CN}$, and $\sigma$ is the standard deviation.

The solvation structures were further analysed by the angles; $\mathrm{N}-$ cation- $\mathrm{N}$ and $\mathrm{O}-$ cation-O for all nitrogen and oxygen atoms within a distance of $0.32 \mathrm{~nm}$ of a cation and their

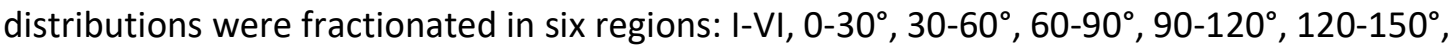
and $150-180^{\circ}$.

\section{Results}

In this study several structural measures of the electrolytes are explored as functions of concentration, cation, and choice of solvent. The RDFs and angular distributions reveal the overall geometry of the solvation structure, while the $\mathrm{CN}$ show its contents and the $\mathrm{CN}$ variance in a general way gauges the diversity of the coordination environments, i.e., high variance is interpreted as numerous stable coordination structures. It should be emphasised that the $\mathrm{CN}$, not the solvation number ( $\mathrm{SN})$, is studied, e.g. $\mathrm{CN}=4$ does not necessarily mean 
4 different ions/molecules coordinating (as some may coordinate bidentately). The RDFs are described in section 3.1, followed by the $\mathrm{CN}$ and its variances in section 3.2 , and finally the angular distributions in section 3.3. Each of the structural measures is described as a function of concentration, first for $\mathrm{Li}^{+}$in $\mathrm{PC}$ and $\mathrm{ACN}$ and then for $\mathrm{Na}^{+}$in $\mathrm{PC}$ and $\mathrm{ACN}$.

\subsection{RDFs}

In the RDFs of the $\mathrm{LiPF}_{6}$ based electrolytes there are clear differences between the two solvents (Fig. 1). The PC based electrolytes (Left panel) show a Li-O peak at $0.21 \mathrm{~nm}$ for all concentrations, in excellent agreement with neutron diffraction data [42], while for the ACN based electrolytes (Right panel), the corresponding Li-N peak is found at $0.22 \mathrm{~nm}$ for the dilute

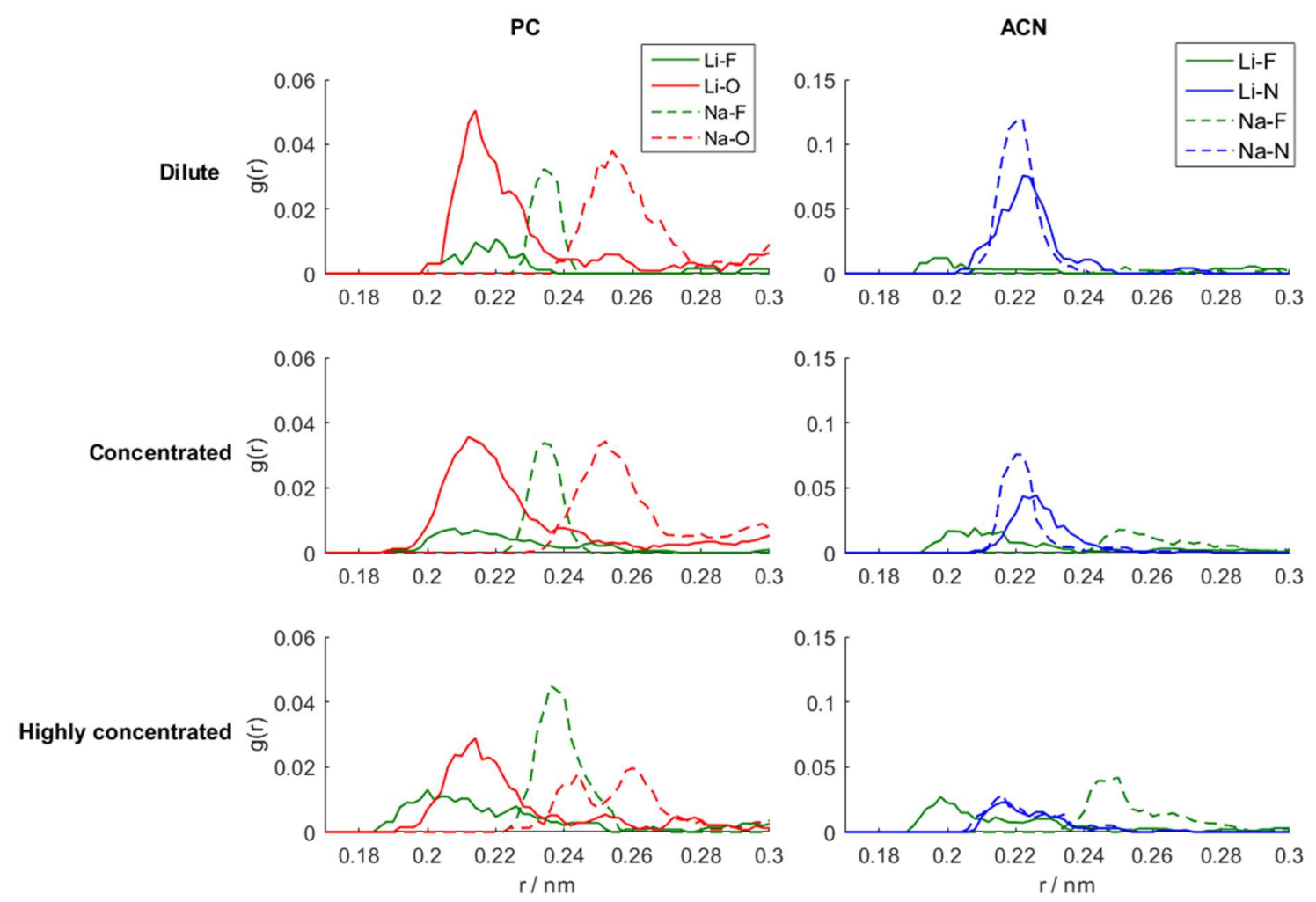

Figure 1. RDF-profiles of the dilute, concentrated, and highly concentrated electrolytes. Solid lines are Li systems and dashed lines Na systems. Left panel shows the PC based electrolytes and right panel ACN based electrolytes. Red display the cation-O, dark green the cation-F, and blue the cation-N RDFs, respectively. 
systems, fairly comparable to diffraction experiments [43]. The latter peak clearly broadens, decreases in intensity, and splits for the highly concentrated systems. The Li-F peaks in the PC based systems overlaps with the Li-O peak in the dilute and concentrated systems, but it broadens and slightly shifts towards lower coordinating distances for the higher concentrations. Interestingly, the Li-F distances are clearly shorter than the Li-N distances for all concentrations in the ACN based electrolytes.

Moving to $\mathrm{Na}^{+}$the RDFs are in stark contrast to the $\mathrm{Li}^{+}$case above - for the $\mathrm{NaPF}_{6}-\mathrm{PC}$ based electrolytes (Fig. 1 - Left panel) both the $\mathrm{Na}-\mathrm{O}$ and $\mathrm{Na}-\mathrm{F}$ peaks are on average $0.03 \mathrm{~nm}$ shifted towards longer distances in comparison with the LiPF 6 -PC cases. In addition, while the $\mathrm{Li}-\mathrm{O}$ and $\mathrm{Li}-\mathrm{F}$ peaks overlap, the $\mathrm{Na}-\mathrm{F}$ distances are clearly shorter than the $\mathrm{Na}-\mathrm{O}$ distances.

Higher salt concentrations in the PC system promotes a split of the $\mathrm{Na}-\mathrm{O}$ peak, in contrast to the unimodal profile observed for $\mathrm{Li}-\mathrm{O}$, suggesting $\mathrm{Na}^{+}$to be less effective in keeping a defined solvation structure. For ACN the $\mathrm{Li}-\mathrm{N}$ and $\mathrm{Na}-\mathrm{N}$ profiles are fairly similar, but increasing the concentration highlights different cation-F distances: the Na-F peak evolves from almost non-existent for the dilute systems, starts to appear in the concentrated systems and then grows to a well-defined peak for the highly concentrated systems. The NaF distances are clearly longer than the Na-N distances, quite the opposite of what is observed for $\mathrm{Li}^{+}$. Indeed, many different computational studies consistently show the $\mathrm{F}$ atoms to coordinate closer to $\mathrm{Li}^{+}$than the lead atom of the solvent, for $\mathrm{ACN}[27,28]$ as well as for EC and DMC [44]. In addition, while the Na-F distances are about $0.02 \mathrm{~nm}$ longer than the Li-F distances in PC electrolytes, they are almost $0.05 \mathrm{~nm}$ longer in $\mathrm{ACN}$ electrolytes, highlighting even more the influence of the solvent on the cation- $\mathrm{F}$ distances. 
In all, increasing the salt concentration promotes a decrease in the cation-solvent peak intensity with a consequent increase in the cation-anion peak intensity. The most pronounced changes occur for the highly concentrated electrolytes, where the split of the Na-O, Li-N and Na-N peaks occur and slight shifts of the Li-F peaks are observed.

\section{2 $\mathrm{CN}$ and $\mathrm{CN}$ variance}

By integrating the first peak in the RDFs the $\mathrm{CNs}$ can be obtained. For the $\mathrm{LiPF}_{6}-\mathrm{PC}$ based systems this results in an averaged solvation structure of 4.3 oxygen and 0.9 fluorine atoms coordinating to a $\mathrm{Li}^{+}$for the dilute systems here denoted $\mathrm{Li}(\mathrm{O})_{4.3}(\mathrm{~F})_{0.9}$. While both mono- or bidentate coordination of $\mathrm{Li}^{+}$by a solvent or anion are possible, these are not separable using only the atomic distances in the RDFs, and there is uncertainty of the exact molecular composition of the solvation structure. However, assuming solely monodentate coordination, $\mathrm{Li}(\mathrm{O})_{4.3}(\mathrm{~F})_{0.9}$ with $\mathrm{CN} \approx 5$ is equal to a $\left[\mathrm{Li}(\mathrm{PC})_{4.3}\left(\mathrm{PF}_{6}\right)_{0.9}\right]$ complex, whereas a $\left[\mathrm{Li}(\mathrm{PC})_{5}\right]^{+}$-complex is reported by both Raman and NMR spectroscopy [45]. For the concentrated systems, the solvation structure evolves to an average of $\mathrm{Li}(\mathrm{O})_{3.4}(\mathrm{~F})_{0.9 \text {. For the }}$ highly concentrated systems the $\mathrm{CN}$ is lowered even further, consisting of $\mathrm{Li}(\mathrm{O})_{2.9}(\mathrm{~F})_{1.8}$ - thus a doubling of the fluorine contribution to the $\mathrm{CN}$ has occurred. For $\mathrm{ACN}$, a $\mathrm{Li}(\mathrm{N})_{3.8}(\mathrm{~F})_{0.7}$ coordination seems to be the most stable at dilute concentrations, moving to $\mathrm{Li}(\mathrm{N})_{3.1}(\mathrm{~F})_{1.8}$ for the concentrated systems, and progressing to a much higher anion content, $\mathrm{Li}(\mathrm{N})_{1.9}(\mathrm{~F})_{2.5}$, for the highly concentrated electrolytes. The total coordination number, however, remains close to 4.5 for all cases, larger than the 4 -fold coordination reported experimentally by different techniques $[43,46-48]$, suggesting the PM7 method to slightly overestimate the coordination number, the cut-off to be set to high, or possibly that the solvent exchange affects the experimental data. As noted above for both the ACN and the PC based 
electrolytes an increased salt concentration promotes a substitution of solvent molecules by anions in the first solvation shell, as expected [28]. The increase of fluorine in the coordination shell can be attributed two effects, an increase in propensity for anions to coordinate the cation when the concentration is increased, and a re-orientation of the anion allowing for higher dentate order as the concentration is increased.

For $\mathrm{Na}^{+}$the $\mathrm{CNs}$ are consistently higher than for $\mathrm{Li}^{+}$for all concentrations. Specifically, in $\mathrm{NaPF}_{6}-\mathrm{PC}$ the solvation structure evolves from $\mathrm{Na}(\mathrm{O})_{4.8}(\mathrm{~F})_{1.5}$ for the dilute electrolytes, to $\mathrm{Na}(\mathrm{O})_{4.0}(\mathrm{~F})_{1.7}$ for the concentrated, and finally $\mathrm{Na}(\mathrm{O})_{2.7}(\mathrm{~F})_{3.6}$ for the highly concentrated electrolytes. In contrast, for the dilute $\mathrm{NaPF}_{6}-\mathrm{ACN}$ electrolytes a $\mathrm{Na}(\mathrm{N})_{4.2}(\mathrm{~F})_{0.7}$ structure is preferred, a $\mathrm{Na}(\mathrm{N})_{3.2}(\mathrm{~F})_{2.8}$ for the concentrated, while $\mathrm{Na}(\mathrm{N})_{2.2}(\mathrm{~F})_{4.8}$ is the most stable configuration for the highly concentrated electrolytes. Thus, a rather monotonic and continuous substitution of solvent for anions in the coordination shell is observed moving from dilute to highly concentrated electrolytes.

Table 1. Coordination numbers and their variance.

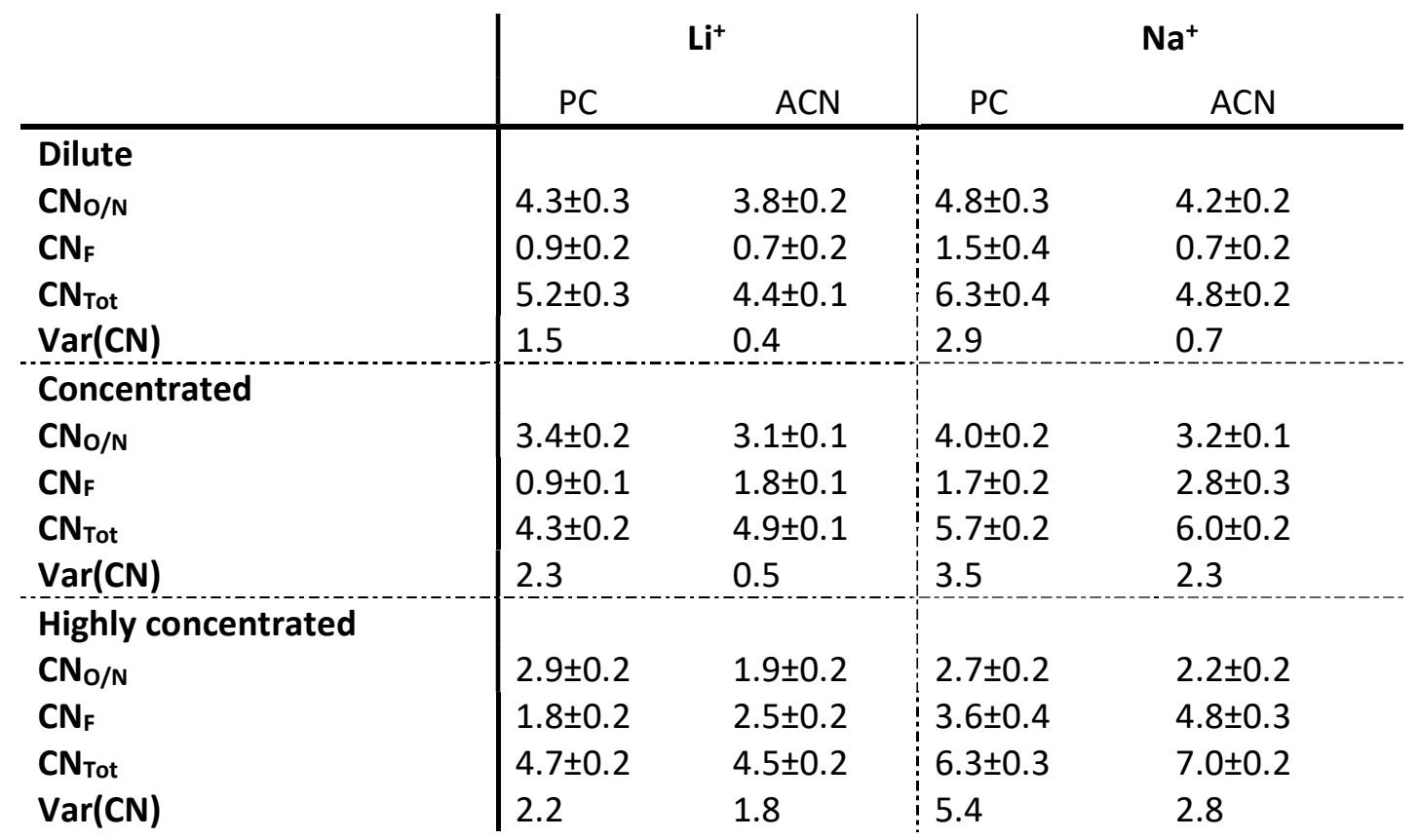


The variance of the $\mathrm{CN}$ in general increases with concentration, but in a different fashion for each type of electrolyte. For the Li-PC and Na-ACN electrolytes the variance has a jump from diluted to concentrated, while for the Li-ACN electrolyte it has a jump from concentrated to highly concentrated, while again for the Na-ACN electrolytes it increases progressively. The PC-based electrolytes possess larger variances for all concentrations as compared to the ACN based electrolytes. In addition, the variances of the ACN based electrolytes remain particularly small for the dilute systems, while the changes with concentration are the most pronounced; almost four times larger for the highly concentrated systems. The variances of the Na-based electrolytes are consistently larger than the Li-based electrolytes. Hence, substituting lithium for sodium and changing solvent from ACN to PC both increase the variance. 


\subsection{Angular distributions}

Starting with the PC-based electrolytes these have an O-cation-O binodal profile, where a broad feature is distributed around $90^{\circ}$ and a sharp peak is centred close to $50^{\circ}$. The latter

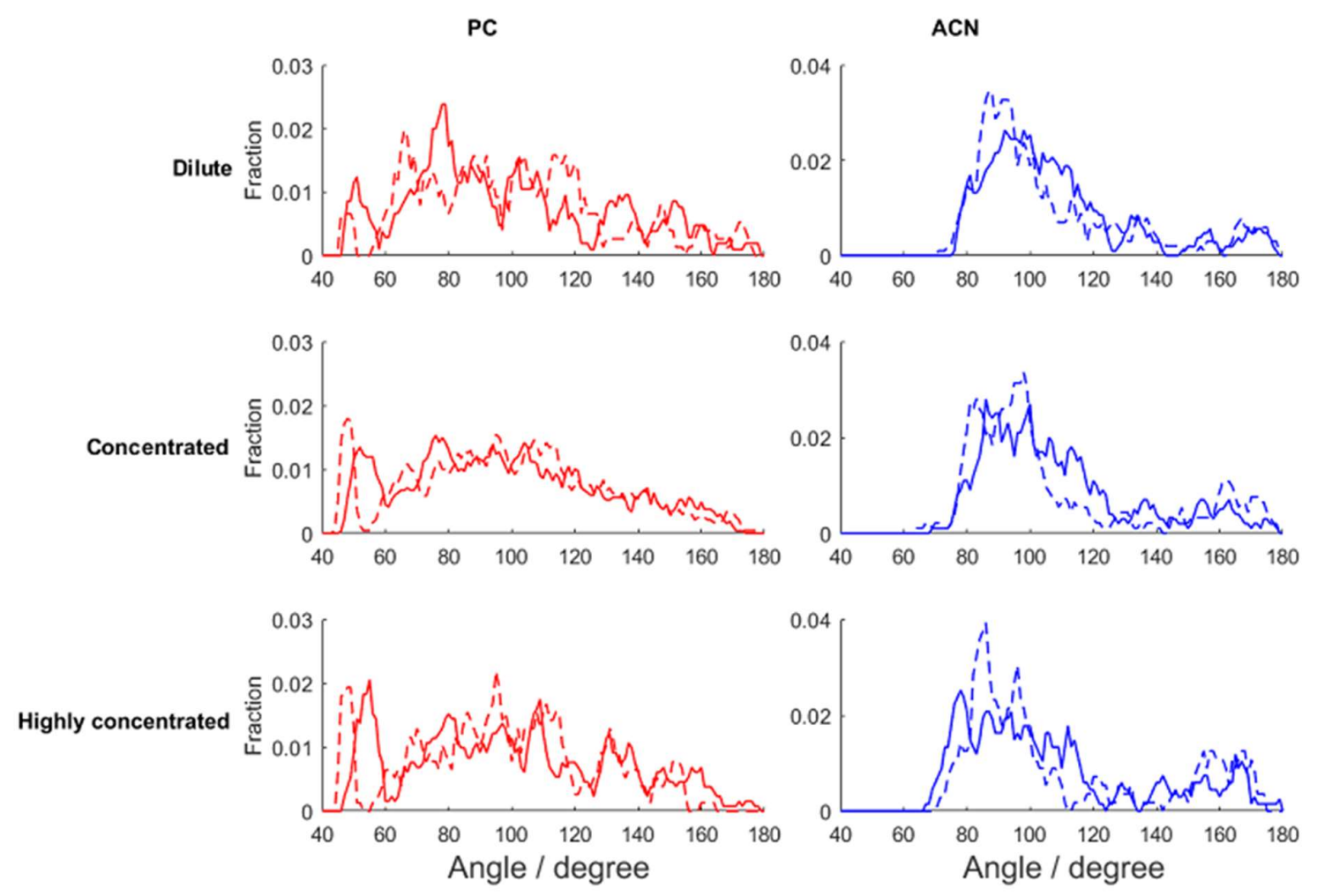

Figure 2. The angle distribution for lithium (solid) and sodium (dashed) in PC and ACN. Left: O-cation-O distribution. Right: $\mathrm{N}-$ cation-N distribution.

strongly suggests a bidentate coordination of the cation (Figs. 2-4 and Table 2) and as the

feature increases with concentration also the bidentate coordination increases with

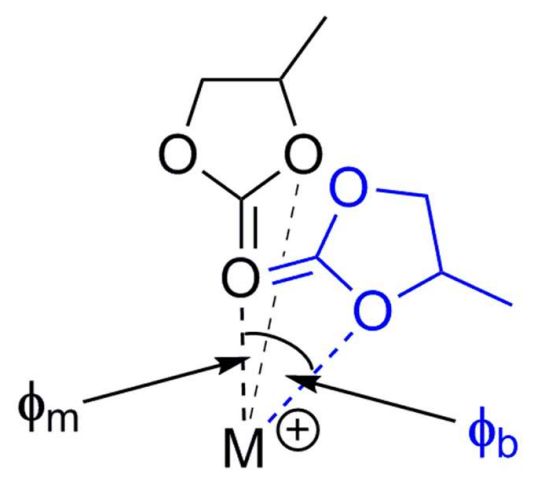

Figure 3. Mono (black) and bidentate coordination (blue) of a cation $\mathrm{M}^{+}$by a single $\mathrm{PC}$ molecule - with the concomitant distance and angle changes. 
concentration, i.e. when increasing the salt concentration it becomes increasingly common that the cation is coordinated both by the carbonyl oxygen atom and an ether oxygen atom belonging to the same PC solvent molecule, hence $\phi_{\mathrm{m}}<\phi_{\mathrm{b}}$ (Fig. 3 and 4).

Simultaneously, the angular distribution becomes more diffuse, in agreement with the increase in the $\mathrm{CN}$ variance. The sharp peak is centred closer to $60^{\circ}$ for the Li-PC case, which indeed is consistent with the RDFs (Fig. 1) where there is a split of the $\mathrm{Na-O}$ peak. The latter indicates that for a bidentate coordination of $\mathrm{Na}^{+}$, the oxygen atoms are situated at different distances, reducing the angle (Fig. 3), whereas for $\mathrm{Li}^{+}$the bidentate coordination is rather of an equilateral triangular shape.

Moving to the ACN based electrolytes these present a binodal $\mathrm{N}-$ cation- $\mathrm{N}$ distribution suggesting a different coordination geometry as compared to the PC based electrolytes.
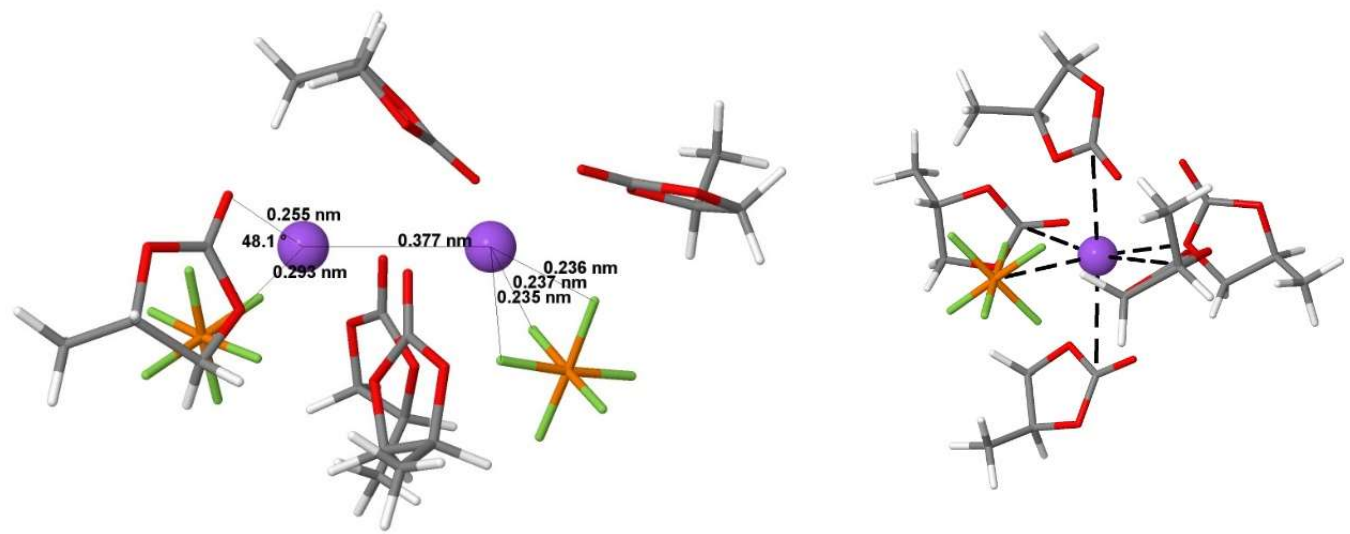

Figure 4. Left: Bidentate coordination by $\mathrm{PC}$ and tridentate coordination by a $\mathrm{PF}_{6}$ anion for $\mathrm{Na}^{+}$with the angles and distances given. Right: An octahedral arrangement of the ligands. The black dashed lines are only guide to the eye.

A single broad peak distributed around $90^{\circ}$, and no feature at $50^{\circ}$, suggests the presence of an equatorial, square-planar alignment of solvent molecules around the cation, a coordination which can also be found in an overall octahedral coordination. In further contrast to the PC based electrolytes, there is a feature at large angles, close to $170^{\circ}$, which indicates a bi-pyramidal, and for solvation shells with a $\mathrm{CN}=6$-octahedral coordination. 
Moreover, there are no large changes occurring when the salt concentration is increased, indicating that no overall structural changes occur in the ACN based electrolytes.

From the more detailed statistics (Table 2), the PC based electrolytes show the fractions of angles within the regions II, IV, and V to increase with salt concentration, and at the same time the fractions in region III decreases, indicating that the shape of the solvation shell is distorted by increasing the salt concentration. The increase in region II indicates that bidentate coordination increases with concentration and in general the large spread from one of the regions into the three others is consistent with the increased $\mathrm{CN}$ variance.

The angles in the ACN based electrolytes mostly fall within regions III and IV. Compared to the PC based electrolytes the distribution is much narrower (Fig. 2), which supports that the $\mathrm{CN}$ variance for the PC based electrolytes is larger than for the ACN based. The fraction of angles in region IV decreases upon increasing the salt concentration, while the angles within regions III and VI become more abundant. Overall the trends seem to be independent of the choice of cation, however, it seems that $\mathrm{Na}^{+}$is more prone to form coordination geometries with $\mathrm{O}-$ cation-O $\mathrm{O}$ and $\mathrm{N}-$ cation- $\mathrm{N}$ angles falling in region IV, while $\mathrm{Li}^{+}$produces a broader distribution of angles.

Table 2. Distribution of $\mathrm{O} / \mathrm{N}-$ cation-O/N angles in the 6 regions corresponding to $0-30^{\circ}, 30-60^{\circ}, 60-90^{\circ}$, $90-120^{\circ}, 120-150^{\circ}$, and $150-180^{\circ}$, respectively.

\begin{tabular}{l|llllll} 
& I & II & III & IV & V & VI \\
\hline LiPF $/ P C$ & & & & & & \\
Dilute & 0.00 & 0.14 & 0.34 & 0.25 & 0.18 & 0.09 \\
Concentrated & 0.00 & 0.18 & 0.29 & 0.29 & 0.17 & 0.07 \\
Highly concentrated & 0.00 & 0.20 & 0.24 & 0.29 & 0.19 & 0.08 \\
\hdashline NaPF 6 /PC & & & & & & \\
Dilute & 0.00 & 0.15 & 0.35 & 0.31 & 0.13 & 0.06 \\
Concentrated & 0.00 & 0.18 & 0.29 & 0.28 & 0.17 & 0.07 \\
Highly concentrated & 0.00 & 0.22 & 0.30 & 0.29 & 0.14 & 0.06 \\
\hdashline LiPF $/$ ACN & & & & & &
\end{tabular}




\begin{tabular}{l|llllll} 
Dilute & 0.00 & 0.00 & 0.30 & 0.46 & 0.14 & 0.10 \\
Concentrated & 0.00 & 0.00 & 0.30 & 0.46 & 0.06 & 0.17 \\
Highly concentrated & 0.00 & 0.00 & 0.37 & 0.31 & 0.10 & 0.22 \\
\hline NaPF,/ACN & & & & & & \\
Dilute & 0.00 & 0.00 & 0.19 & 0.60 & 0.12 & 0.09 \\
Concentrated & 0.00 & 0.00 & 0.24 & 0.53 & 0.12 & 0.11 \\
Highly concentrated & 0.00 & 0.00 & 0.36 & 0.41 & 0.10 & 0.14
\end{tabular}

\section{Discussion}

\subsection{Solvation structure changes}

The electrolyte structure in terms of the cation first solvation shell is quite clearly influenced by concentration, solvent, and choice of cation. Increasing the salt concentration promotes the split in the RDFs (Fig. 1), with a higher proportion of fluorine atoms, i.e., anions in the first coordination shell and an increase in the $\mathrm{CN}$ variance (Table 1). At low concentrations and for highly dissociated salts like LiPF $_{6}$ the abundance of solvent molecules is enough to stabilize the first solvation shell of the cations via lone-pair coordination and are predominant. Since these Lewis-type acid-base interactions are highly directional, the solvation structure is expected to be well-defined. At high salt concentrations solvent molecules are scarce and not enough to stabilize the cation's positive charge. Thus, anions are expected to contribute more to the charge stabilization via coulombic ion-ion interactions, as reflected in the increased proportion of fluorine atoms in the coordination shell. Since the anion's negative charge is delocalized, the coordination structure is expected to be less defined and more flexible at high concentrations, as reflected by the broad RDFs and the increased variances.

As for the influence of the cation, the $\mathrm{Na}^{+}$containing electrolytes in general exhibit longer cation-solvent and cation-anion distances (Fig. 1), higher CNs, larger $\mathrm{CN}$ variance, and a 
larger proportion of fluorine atoms in the first solvation shell (Table 1). Thus, compared to $\mathrm{LiPF}_{6}$, the $\mathrm{NaPF}_{6}$ based electrolytes have larger, more populated and disordered coordination shells. From a chemical perspective, $\mathrm{Na}^{+}$and $\mathrm{Li}^{+}$differ mainly by their ionic radii $\left(\mathrm{r}_{\mathrm{Na}+} \approx 0.1\right.$ $\mathrm{nm}, \mathrm{r}_{\mathrm{Li}+} \approx 0.06 \mathrm{~nm}$ ) [49]. Indeed, the larger coordination distances are likely related to the cation size as the RDFs shows the Na-solvent and Na-F distances to be 0.02 to $0.05 \mathrm{~nm}$ larger than the corresponding distances in Li-based electrolytes, in close agreement with the differences in ionic radius. In addition, Pearson and Klopman's principles based on chemical hardness [50] predict $\mathrm{Na}^{+}$, a bigger cation and consequently less hard than $\mathrm{Li}^{+}$, to be more affine to interact with a chemically soft anion such as $\mathrm{PF}_{6}^{-}$, thus explaining the increased presence of fluorine atoms in the first solvation shell of $\mathrm{Na}^{+}$.

Finally, the ACN based electrolytes display an angular distribution that resembles octahedral cation coordination, different from the coordination geometry observed in the PC based electrolytes. In addition, the PC based electrolytes show larger CN variance and longer cation-solvent distances. Since PC and ACN both have comparable dielectric constants and donor numbers, these differences likely arises from the number of possible coordination sites per solvent molecule, i.e., PC allows for bidentate coordination as seen in the angular distribution and in Figs. 3 and 4.

\subsection{Practical consequences for battery usage}

The ionic conductivity is essentially known to decrease with increased salt concentration, for all except very dilute systems, and this should be detrimental for the applicability of highly concentrated electrolytes. However, increases in the cation transference number has been observed in ionic liquids [23-25], and increased lithium conductivity [18], indicating that 
while the overall conductivity decreases the crucial cationic conductivity might even increase when the salt concentration is elevated beyond the conventional, concentrated, levels.

For dilute systems the ionic conductivity mainly occurs via a vehicular mechanism where the conductivity strongly depends on the size of the charge carrier and the electrolyte viscosity [51]. For these systems the $\mathrm{Li}^{+}$based electrolytes are expected to have higher conductivities than the $\mathrm{Na}^{+}$based electrolytes due to a denser and likely smaller solvation shell (hence diffusing faster). In contrast, MD simulations show that the extensive ion-ion interactions present for higher salt concentrations promote electrolyte structures that resemble polymeric highly viscous networks, where cations have short residence times at their coordination sites $[30,44]$. Under such conditions the ionic conductivity might follow a Grotthuss-like transport mechanism, where cations move via a sequential series of jumps between neighbouring sites and the ionic conductivity is decoupled from the viscosity $[23,24,52]$. High CN variance, here interpreted as numerous stable structures, seems to reflect the flexible character of coordination sites for the higher salt concentrations predicted by the MD simulations. Moreover, for the high salt concentrations the scarcity of solvent molecules forces the cations to compete for the contents of their solvation shell, and hence the solvation shells formed are likely energetically less stable than at lower concentrations where the cation is able to form its energetically preferred shell. Again, this gives credence to that at high concentrations, a high variance and a reduction in the residence time results. In this context the $\mathrm{Na}^{+}$based electrolytes, with higher $\mathrm{CN}$ variance, are expected to present more of this behaviour than the $\mathrm{Li}^{+}$based electrolytes.

The liquid range of the electrolyte is another key parameter for LIB and SIB performance, since the electrolyte should remain in the liquid state in order to keep the ionic conductivity 
practical and to wet the porous electrodes [12,51]. A fundamental description of the liquidus range can be obtained via an analysis of the potential energy surface (PES) as for glassforming liquids there is a relationship between the number of accessible PES minima at a given temperature and the system relaxation time, increasing significantly when approaching the glass transition temperature. In essence, a liquid with a reduced number of accessible PES minima (reduced number of configurations) tend to be viscous and easily evolves to a structure with solid-like properties [53]. Based on this argument, electrolytes with numerous stable structural configurations are expected to have an improved liquid range, i.e., to remain liquid for a wider range of temperatures and salt concentrations. Therefore, the electrolytes with higher variance at high concentrations can be anticipated to have a wide liquid ranges. Here this implies that the $\mathrm{Na}^{+}-\mathrm{PC}$ based electrolytes remain liquid for higher concentrations and lower temperatures than the $\mathrm{Li}^{+}-\mathrm{ACN}$ based electrolytes. This is partially supported by the liquid ranges of $1.0 \mathrm{M} \mathrm{NaPF}_{6}-\mathrm{PC}$, remaining liquid down to $-95^{\circ} \mathrm{C}$ [54], and 1.0 $\mathrm{M} \mathrm{LiPF}_{6}-\mathrm{ACN}$, crystallizing already at room temperature [30]. In this sense, the analysis of the $\mathrm{CN}$ variance represents a tool to link the local molecular picture obtained from quantum mechanical computations with macro-scale battery relevant electrolyte properties.

A third property of practical importance is the cation de-solvation energetics and kinetics to reduce charge transfer and interfacial resistance. Xu, Ogumi and co-workers demonstrated that an energetically demanding cation de-solvation significantly decreased the charge transfer kinetics $[20,21]$. An increased proportion of anions in the cation solvation shell could facilitate cation de-solvation at the anode surface, as the negatively charged electrode should repel anions, creating a de-stabilized solvation structure where the solvent molecules are no longer sterically hindered from re-arranging. In principle, this should allow 
the cation to more easily strip out of its shell. In this context, highly concentrated electrolytes, observed to have cations with an increased proportion of fluorine atoms, i.e., anions, in their solvation shell, are expected to have improved kinetics. This could also explain why enhanced kinetics mainly have been reported for negative electrodes $[13,18,19,55]$.

\section{Conclusions}

For $\mathrm{Li}^{+}$and $\mathrm{Na}^{+}-\mathrm{PC}$ and $\mathrm{ACN}$ based electrolytes the RDFs obtained from our PM7 computations are able to in detail reveal the solvation structures and these can be linked to several properties of practical importance for LIB and SiB electrolytes. In general, the RDFs become more disordered for the higher salt concentrations as compared to the dilute systems and the sodium cations have longer solvent and anion coordination distances as compared to the lithium cations. The cation $\mathrm{CNs}$ and $\mathrm{CN}$ variance show salt concentration to increase the population and the proportion of fluorine atoms in the first solvation shell, both these trends are particularly pronounced for the $\mathrm{Na}^{+}$based electrolytes, and the latter suggesting numerous stable structures i.e. flexible first solvation shells. The angular distributions have less of a concentration dependence, but differ substantially between ACN and PC electrolytes, suggesting the choice of solvent to be decisive for the cation coordination geometry. The scarcity of solvent molecules and the extensive ion-ion interactions that occur at high salt concentrations affect the concentration dependent trends, while the differences observed between the $\mathrm{Li}^{+}$and $\mathrm{Na}^{+}$based electrolytes are primarily attributed to the different cation ionic radii and their chemical hardness. In addition, the $\mathrm{Na}^{+}-\mathrm{PC}$ based electrolytes should have improved liquid ranges at high concentrations due to highly flexible coordination structures. Moreover, the highly 
concentrated electrolytes should also display relatively faster charge transfer kinetics, as indeed observed experimentally at negative electrodes, a feature linked to a cation coordination structure with a pronounced presence of anions.

\section{Acknowledgements}

We acknowledge the continuous support from several of Chalmers Areas of Advance: Materials Science, Energy, and Transport. GÅ and PJ also acknowledges the EU H2O20 NAIADES project (LCE10-2014, \#646433) and the support from the Swedish Energy Agency through "Batterifondsprogrammet": Next Generation Batteries (\#37671-1). This project was initiated as a master thesis (EF) performed mainly at Chalmers within the Eramus Mundus MESC programme hosted at Univ. Picardie Jules Verne at Amiens, France, by Alistore-ERI.

\section{References}

[1] A. Ponrouch, D. Monti, A. Boschin, B. Steen, P. Johansson, M.R. Palacín, Non-aqueous electrolytes for sodium-ion batteries, J. Mater. Chem. A. 3 (2015) 22-42. doi:10.1039/C4TA04428B.

[2] E. Karden, S. Ploumen, B. Fricke, T. Miller, K. Snyder, Energy storage devices for future hybrid electric vehicles, J. Power Sources. 168 (2007) 2-11. doi:10.1016/j.jpowsour.2006.10.090.

[3] 2014 Minerals Yearbook. http://minerals.usgs.gov/minerals/pubs/commodity/lithium/myb1-2014-lithi.pdf.

[4] 2015 Minerals Yearbook. http://minerals.usgs.gov/minerals/pubs/commodity/soda_ash/myb1-2015-sodaa.pdf.

[5] J.-M. Tarascon, M. Armand, Issues and challenges facing rechargeable lithium 
batteries, Nature. 414 (2001) 359-367. doi:10.1038/35104644.

[6] A. Ponrouch, R. Dedryvère, D. Monti, A.E. Demet, J.M. Ateba Mba, L. Croguennec, C. Masquelier, P. Johansson, M.R. Palacín, Towards high energy density sodium ion batteries through electrolyte optimization, Energy Environ. Sci. 6 (2013) 2361-2369. doi:10.1039/c3ee41379a.

[7] D. Larcher, J.-M. Tarascon, Towards greener and more sustainable batteries for electrical energy storage, Nat. Chem. 7 (2014) 19-29. doi:10.1038/nchem.2085.

[8] H. Pan, Y.-S. Hu, L. Chen, Room-temperature stationary sodium-ion batteries for largescale electric energy storage, Energy Environ. Sci. 6 (2013) 2338. doi:10.1039/c3ee40847g.

[9] K. Kubota, S. Komaba, Review-Practical Issues and Future Perspective for Na-Ion Batteries, J. Electrochem. Soc. 162 (2015) A2538-A2550. doi:10.1149/2.0151514jes.

[10] K. Xu, Nonaqueous Liquid Electrolytes for Lithium-Based Rechargeable Batteries, Chem. Rev. 104 (2004) 4303-4418. doi:10.1021/cr030203g.

[11] S. Tan, Y.J. Ji, Z.R. Zhang, Y. Yang, Recent progress in research on high-voltage electrolytes for lithium-ion batteries, ChemPhysChem. 15 (2014) 1956-1969. doi:10.1002/cphc.201402175.

[12] T.R. Jow, M.S. Ding, K. Xu, S.S. Zhang, J.L. Allen, K. Amine, G.L. Henriksen, Nonaqueous electrolytes for wide-temperature-range operation of Li-ion cells, J. Power Sources. 119-121 (2003) 343-348. doi:10.1016/S0378-7753(03)00153-8.

[13] Y. Yamada, K. Furukawa, K. Sodeyama, K. Kikuchi, M. Yaegashi, Y. Tateyama, A. Yamada, Unusual Stability of Acetonitrile-Based Superconcentrated Electrolytes for 
Fast-Charging Lithium-Ion Batteries, J. Am. Chem. Soc. 136 (2014) 5039-5046.

[14] K. Xu, Electrolytes and interphases in Li-ion batteries and beyond, Chem. Rev. 114 (2014) 11503-11618. doi:10.1021/cr500003w.

[15] J.Y. Song, Y.Y. Wang, C.C. Wan, Review of gel-type polymer electrolytes for lithium-ion batteries, J. Power Sources. 77 (1999) 183-197. doi:10.1016/S0378-7753(98)00193-1.

[16] Y. Yamada, A. Yamada, Review-Superconcentrated Electrolytes for Lithium Batteries, J. Electrochem. Soc. 162 (2015) A2406-A2423. doi:10.1149/2.0041514jes.

[17] Y. Yamada, K. Usui, C.H. Chiang, K. Kikuchi, K. Furukawa, A. Yamada, General Observation of Lithium Intercalation into Graphite in Ethylene-Carbonate-Free Superconcentrated Electrolytes, ACS Appl. Mater. Interfaces. 6 (2014) 10892-10899. doi:10.1021/am5001163.

[18] L. Suo, Y.-S. Hu, H. Li, M. Armand, L. Chen, A new class of Solvent-in-Salt electrolyte for high-energy rechargeable metallic lithium batteries, Nat. Commun. 4 (2013) 1481. doi:10.1038/ncomms2513.

[19] Y. Yamada, M. Yaegashi, T. Abe, A. Yamada, A superconcentrated ether electrolyte for fast-charging Li-ion batteries, Chem. Commun. 49 (2013) 11194-11196. doi:10.1039/C3CC46665E.

[20] T. Abe, H. Fukuda, Y. Iriyama, Z. Ogumi, Solvated Li-Ion Transfer at Interface Between Graphite and Electrolyte, J. Electrochem. Soc. 151 (2004) A1120-A1123. doi:10.1149/1.1763141.

[21] K. Xu, A. von Wald Cresce, $\mathrm{Li}^{+}$-solvation/desolvation dictates interphasial processes on graphitic anode in Li ion cells, J. Mater. Res. 27 (2012) 2327-2341. 
doi:10.1557/jmr.2012.104.

[22] D.W. McOwen, D.M. Seo, O. Borodin, J. Vatamanu, P.D. Boyle, W.A. Henderson, Concentrated electrolytes: decrypting electrolyte properties and reassessing $\mathrm{Al}$ corrosion mechanisms, Energy Environ. Sci. 7 (2014) 416-426.

doi:10.1039/C3EE42351D.

[23] M. Forsyth, H. Yoon, F. Chen, H. Zhu, D. R. MacFarlane, M. Armand, P.C. Howlett, Novel $\mathrm{Na}^{+}$Ion Diffusion Mechanism in Mixed Organic-Inorganic Ionic Liquid Electrolyte Leading to High $\mathrm{Na}^{+}$Transference Number and Stable, High Rate Electrochemical Cycling of Sodium Cells., J. Phys. Chem. C. 120 (2016) 4276-4286. doi:10.1021/acs.jpcc.5b11746.

[24] F. Chen, M. Forsyth, Elucidation of transport mechanism and enhanced alkali ion transference numbers in mixed alkali metal-organic ionic molten salts, Phys. Chem. Chem. Phys. 18 (2016) 19336-19344. doi:10.1039/C6CP01411A.

[25] K. Matsumoto, Y. Okamoto, T. Nohira, R. Hagiwara, Thermal and Transport Properties of $\mathrm{Na}\left[\mathrm{N}\left(\mathrm{SO}_{2} \mathrm{~F}\right)_{2}\right]-\left[\mathrm{N}-\right.$ Methyl-N-propylpyrrolidinium] $\left[\mathrm{N}\left(\mathrm{SO}_{2} \mathrm{~F}\right)_{2}\right]$ lonic Liquids for $\mathrm{Na}$ Secondary Batteries, J. Phys. Chem. C. 119 (2015) 7648-7655. doi:10.1021/acs.jpcc.5b01373.

[26] S.K. Jeong, M. Inaba, Y. Iriyama, T. Abe, Z. Ogumi, Interfacial reactions between graphite electrodes and propylene carbonate-based solutions: Electrolyteconcentration dependence of electrochemical lithium intercalation reaction, J. Power Sources. 175 (2008) 540-546. doi:10.1016/j.jpowsour.2007.08.065.

[27] D.M. Seo, O. Borodin, S.-D. Han, Q. Ly, P.D. Boyle, W.A. Henderson, Electrolyte 
Solvation and Ionic Association, J. Electrochem. Soc. 159 (2012) A553. doi:10.1149/2.jes112264.

[28] D.M. Seo, O. Borodin, S.-D. Han, P.D. Boyle, W.A. Henderson, Electrolyte Solvation and Ionic Association II. Acetonitrile-Lithium Salt Mixtures: Highly Dissociated Salts, J. Electrochem. Soc. 159 (2012) A1489-A1500. doi:10.1149/2.035209jes.

[29] D. Spångberg, K. Hermansson, The solvation of $\mathrm{Li}^{+}$and $\mathrm{Na}^{+}$in acetonitrile from ab initio-derived many-body ion-solvent potentials, Chem. Phys. 300 (2004) 165-176. doi:10.1016/j.chemphys.2004.01.011.

[30] D.M. Seo, O. Borodin, D. Balogh, M. O'Connell, Q. Ly, S.-D. Han, S. Passerini, W.A. Henderson, Electrolyte Solvation and Ionic Association III. Acetonitrile-Lithium Salt Mixtures-Transport Properties, J. Electrochem. Soc. 160 (2013) A1061-A1070. doi:10.1149/2.018308jes.

[31] K. Sodeyama, Y. Yamada, K. Aikawa, A. Yamada, Y. Tateyama, Sacrificial Anion Reduction Mechanism for Electrochemical Stability Improvement in Highly Concentrated Li-Salt Electrolyte, J. Phys. Chem. C. 118 (2014) 14091-14097. doi:10.1021/jp501178n.

[32] J.J.P. Stewart, Optimization of parameters for semiempirical methods VI: more modifications to the NDDO approximations and re-optimization of parameters, J. Mol. Model. 19 (2013) 1-32. doi:10.1007/s00894-012-1667-x.

[33] J. Hostaš, J. Řezáč, P. Hobza, On the performance of the semiempirical quantum mechanical PM6 and PM7 methods for noncovalent interactions, Chem. Phys. Lett. 568-569 (2013) 161-166. doi:10.1016/j.cplett.2013.02.069. 
[34] A. Rybinska, A. Sosnowska, M. Barycki, T. Puzyn, Geometry optimization method versus predictive ability in QSPR modeling for ionic liquids, J. Comput. Aided. Mol. Des. 30 (2016) 165-176. doi:10.1007/s10822-016-9894-3.

[35] T. Puzyn, N. Suzuki, M. Haranczyk, J. Rak, Calculation of Quantum-Mechanical Descriptors for QSPR at the DFT Level: Is It Necessary?, J. Chem. Inf. Model. 48 (2008) 1174-1180. doi:10.1021/ci800021p.

[36] A. Sosnowska, M. Barycki, M. Zaborowska, A. Rybinska, T. Puzyn, Towards designing environmentally safe ionic liquids: the influence of the cation structure, Green Chem. 16 (2014) 4749-4757. doi:10.1039/C4GC00526K.

[37] V. Chaban, Solvation of lithium ion in dimethoxyethane and propylene carbonate, Chem. Phys. Lett. 631-632 (2015) 1-5. doi:10.1016/j.cplett.2015.04.047.

[38] V. Chaban, Solvation of the fluorine containing anions and their lithium salts in propylene carbonate and dimethoxyethane, J. Mol. Model. 21 (2015) 172. doi:10.1007/s00894-015-2717-y.

[39] M.D. Hanwell, D.E. Curtis, D.C. Lonie, T. Vandermeerschd, E. Zurek, G.R. Hutchison, Avogadro: An advanced semantic chemical editor, visualization, and analysis platform, J. Cheminform. 4 (2012) 1-17. doi:10.1186/1758-2946-4-17.

[40] MOPAC2012, James J. P. Stewart, Stewart Computational Chemistry, Version 15.321W web: HTTP://OpenMOPAC.net

[41] J.D.C. Maia, G.A. Urquiza Carvalho, C.P. Mangueira, S.R. Santana, L.A.F. Cabral, G.B. Rocha, GPU Linear Algebra Libraries and GPGPU Programming for Accelerating MOPAC Semiempirical Quantum Chemistry Calculations, J. Chem. Theory Comput. 8 
(2012) 3072-3081. doi:10.1021/ct3004645.

[42] Y. Kameda, Y. Umebayashi, M. Takeuchi, M.A. Wahab, S. Fukuda, S.-I. Ishiguro, M. Sasaki, Y. Amo, T. Usuki, Solvation Structure of $\mathrm{Li}^{+}$in Concentrated $\mathrm{LiPF}_{6}$-Propylene Carbonate Solutions, J. Phys. Chem. B. 111 (2007) 6104-6109. doi:10.1021/jp072597b.

[43] T. Cartaillert, W. Kunzt, P. Turq, M.-C. Bellisent-Funel, Lithium bromide in acetonitrile and water: a neutron scattering study, J. PHYSICS-CONDENSED MATTER. 3 (1991) 9511-9520.

[44] O. Borodin, G.D. Smith, Quantum Chemistry and Molecular Dynamics Simulation Study of Dimethyl Carbonate: Ethylene Carbonate Electrolytes Doped with $\mathrm{LiPF}_{6}, \mathrm{~J}$. Phys. Chem. B. 113 (2009) 1763-1776. doi:10.1021/jp809614h.

[45] K. Kondo, M. Sano, A. Hiwara, T. Omi, M. Fujita, A. Kuwae, M. lida, K. Mogi, H. Yokoyama, Conductivity and Solvation of $\mathrm{Li}^{+}$Ions of $\mathrm{LiPF}_{6}$ in Propylene Carbonate Solutions, J. Phys. Chem. B. 104 (2000) 5040-5044. doi:10.1021/jp000142f.

[46] A.K. Mollner, W.R. Fawcett, P.A. Brooksby, J.S. Loring, A Look at lon - Solvent Interactions in Acetonitrile, (2015) 1-8.

[47] H.J. Gores, J.M.G. Barthel, Nonaqueous electrolyte solutions: New materials for devices and processes based on recent applied research, Pure Appl. Chem. 67 (1995) 919-930. doi:10.1351/pac199567060919.

[48] L.A. Solovieva, S.K. Akopyan, E. Vilaseca, Determination of ion association constants by means of IR absorption band intensities of the molecules forming the first coordination spheres of the cations, Spectrochim. Acta Part A Mol. Spectrosc. 50 
(1994) 683-688. doi:10.1016/0584-8539(94)80004-9.

[49] R.D. Shannon, Revised effective ionic radii and systematic studies of interatomic distances in halides and chalcogenides, Acta Crystallogr. Sect. A. A32 (1976) 751-767. doi:10.1107/S0567739476001551.

[50] G. Klopman, Chemical reactivity and the concept of charge- and frontier-controlled reactions, J. Am. Chem. Soc. 90 (1968) 223-234. doi:10.1021/ja01004a002.

[51] K. Xu, M. About, T. Article, Nonaqueous Liquid Electrolytes for Lithium-Based Rechargeable Batteries Nonaqueous Liquid Electrolytes for Lithium-Based Rechargeable Batteries, Chem. Rev. 104 (2004) 4303-4418. doi:10.1021/cr030203g.

[52] N. Agmon, The Grotthuss mechanism, Chem. Phys. Lett. 244 (1995) 456-462. doi:10.1016/0009-2614(95)00905-J.

[53] P.G. Debenedetti, F.H. Stillinger, Supercooled liquids and the glass transition, Nature. 410 (2001) 259-267. doi:10.1038/35065704.

[54] A. Ponrouch, E. Marchante, M. Courty, J.-M. Tarascon, M.R. Palacín, In search of an optimized electrolyte for Na-ion batteries, Energy Environ. Sci. 5 (2012) 8572. doi:10.1039/c2ee22258b.

[55] J. Qian, W.A. Henderson, W. Xu, P. Bhattacharya, M. Engelhard, O. Borodin, J.-G. Zhang, High rate and stable cycling of lithium metal anode, Nat. Commun. 6 (2015) 6362. doi:10.1038/ncomms7362. 